\title{
Conductor Bundle Geometry Optimization in View of Conductor Surface Gradient
}

\author{
Member Jong Keun Park (Seoul National University) \\ Non-member Suk Won Min (Soon Chun Hyang University) \\ Non-member Jeong Boo Kim (Korea Electric Power Corp.)
}

This paper describes a technique on conductor bundle geometry optimization by the use of personal computer. To reduce the corona noise, in this paper, a new method for finding the optimal arrangement of subconductors with the minimum surface gradient is proposed. The charge simulation method is used to calculate the electric field of conductor surface and the nonlinear successive quadratic programming is applied to find the optimal arrangement of subconductors. Through some analytical comparisons and corona cage tests, it is proved that a new asymmetric conductor bundle proposed in this paper can more reduce the average surface gradient of the phase conductor and the corona noise than the asymmetric conductor bundle proposed by GE as well as the conventional symmetric ones.

Key words : Corona noise, Asymmetric conductor bundle, Conductor surface gradient, Charge simulation method, Nonlinear successive quadratic programming

\section{Introduction}

The bulk power transmission line over the long distance is introduced to reduce the transmission cost and to use the right of way efficiently. To increase the transmission capacity at lower cost, adoption of the higher voltage transmission system is reasonable in view of the technology and the economy.

However, if the transmission voltage is above 500 $\mathrm{kV}$, the conductor size is often determined by corona performance as well as current capacity and conductor bundle configurations are usually necessary to meet the corona interference such as AN (Audible Noise), RI (Radio Interference), TVI (Television Interference). The corona interference in $\mathrm{AC}$ transmission occurs primarily in foul weather. In wet conditions, however, water drops impinging or collecting on the conductors produce a large number of corona discharges when the electric field intensity exceeds the corona onset value.
Therefore, a key for the reduction of the corona interference is to lower the conductor surface gradient $^{(2)}$.

The general method for reducing the corona noise is the symmetric arrangement of subconductors on the circle with a constant radius to increase the equivalent bundle diameter. In those papers, the determination of the subconductor number, diameter and spacing is the main concern ${ }^{(2)-15)}$.

Meanwhile, conductor bundle optimization in view of corona noise was tried by Mr. Comber and Mr. Zaffanella in the GE (General Electric). They found that the asymmetric conductor bundle could more reduce the corona noise than the symmetric one through the corona cage test ${ }^{(4)}$. To reduce the corona noise of the bottom subconductors, they determined the mutual relation of each subconductor spacing, which can equalize the corona noise of each subconductor. However, the conductor bundle had a drawback to increase the upper subconductors gradient reversely. 
In this study, to reduce the corona noise of each subconductor, a new asymmetric conductor bundle with the minimum surface gradient is determined by the use of nonlinear successive quadratic programming with the variable of each subconductor position. To verify the propriety of this method, corona characteristics on symmetric conductor bundle, asymmetric conductor bundle proposed by $\mathrm{GE}$, and asymmetric conductor bundle proposed in this paper is investigated through the corona cage test.

\section{Geometry optimization method}

\subsection{GE Method}

In a regular bundle, the subconductors generate different amounts of audible noise. The bottom subconductors are noisier than the upper subconductors. This is apparent by visual observations of bundles in corona during foul weather nights.

Accordingly, to reduce the corona noise of the bottom subconductors and to equalize the corona noise of each subconductor, $\mathrm{GE}$ developed the asymmetric conductor bundle in which the subconductors lie on a circle, and the circumferential distances between adjacent subconductors are in geometric proportion $^{(4)(6)}$.

For example, the 6-conductor bundle shown in Fig. 1 complied with these restrictions;

$$
\Delta_{2} / \Delta_{1}=\Delta_{3} / \Delta_{2}=\Delta_{4} / \Delta_{3}
$$

Then the subconductor positions relative to each other were completely derived by the bundle diameter $(D)$ and the parameter $E_{0}=\Delta_{4} / \Delta_{1}$ which might be defined as the degree of asymmetry. Based on

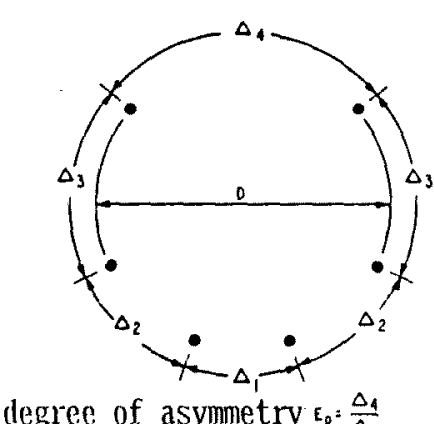

Fig. 1. GE asymmetric arrangement of 6 conductor bundle. these data, the asymmetric conductor bundle was determined through corona cage test which could give minimum corona interference. According to their study, corona interference was minimum when the value of $E_{0}$ was between 2 and $3^{(4)(6)}$. But this asymmetric conductor bundle had a drawback to increase the electric field intensity of the upper subconductors.

\subsection{Method proposed in this paper}

The subconductors in the method of GE were moved on a circle with a constant radius, but in this method the subconductors are moved in the positions which the surface gradient of the lowest phase conductor bundle becomes minimum by the use of charge simulation method and nonlinear successive quadratic programming ${ }^{(7) \sim(9)}$. The objective func. tion $L$, which minimizes the surface gradient of the lowest phase conductor bundle in the three-phase double circuit transmision line as shown in Fig. 2, can be formulated using the charge simulation method. If the unknown infinite line charge is positioned at the center of each subconductor $\left(X_{j}\right.$, $Y_{j}$ ) and the calculation point on the surface of each subconductor $\left(x_{1 k}, y_{1 k}\right)$ is fixed, the electric field intensity at the calculation point can be represented as follows.

$$
\begin{aligned}
F_{i k x}= & \sum_{j=1}^{s} \frac{1}{2 \pi \varepsilon_{0}}-\left(x_{1 k}-X_{j}\right)\left(Q_{j R}^{2}+Q_{j i m}^{2}\right)^{1 / 2} \\
& \times\left\{\frac{1}{\left(x_{i k}-X_{j}\right)^{2}+\left(y_{1 k}-Y_{j}\right)^{2}}\right. \\
& \left.-\frac{1}{\left(x_{1 k}-X_{j}\right)^{2}+\left(y_{1 k}+Y_{j}\right)^{2}}\right\}
\end{aligned}
$$




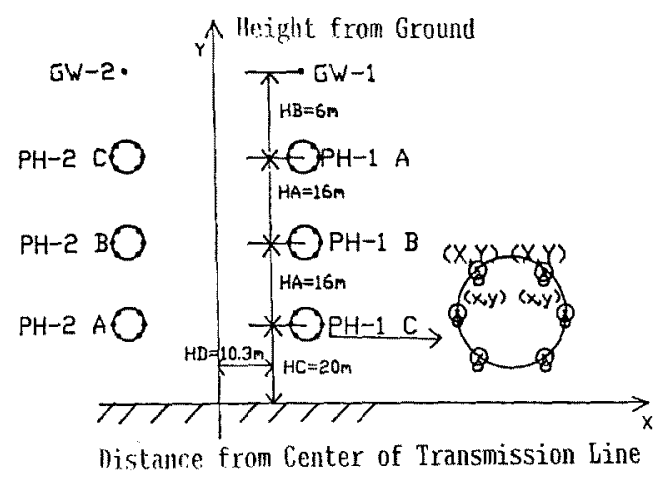

Fig. 2. Configuration of $765 \mathrm{kV}$ double circuit transmission line.

$y_{1 k}: Y$ coordinate of the calculation points

$X_{j}: X$ coordinate of the infinite line charge points

$Y_{j}: Y$ coordinate of the infinite line charge points

$Q_{j R}:$ real part of the infinite line charge quantity

$Q_{i i m}:$ imaginary part of the infinite line charge quantity

$S$ : number of the infinite line charge

After all, the objective function $L$ can be represented as follows.

Objective function $L$ :

$$
L=\min \frac{1}{m n} \sum_{l=1}^{m} \sum_{k=1}^{n}\left(F_{1 k x}^{2}+F_{1 k y}^{2}\right)^{1 / 2}
$$

where

$m$ : number of subconductors per phase conductor bundle

$n$ : number of the calculation points at each subconductor surface

Constraint can be also represented by the use of charge simulation method. If the contour points on the surface of each subconductor $\left(x_{i}, y_{i}\right)$ with a known potential are fixed as the numbers $(S)$ of the infinite line charge position $\left(X_{j}, Y_{j}\right)$, Maxwell potential coefficient $\left(P_{i j}\right)$ between the charge points and contour points is as follows.

$$
P_{i j}=\frac{1}{4 \pi \varepsilon_{0}} \ln \frac{\left(x_{i}-X_{j}\right)^{2}+\left(y_{i}+Y_{j}\right)^{2}}{\left(x_{i}-X_{j}\right)^{2}+\left(y_{i}-Y_{j}\right)^{2}}
$$

where

$x_{i}: X$ coordinate of contour points

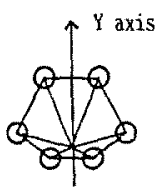

Fig. 3. Asymmetric arrangement proposed in this paper.

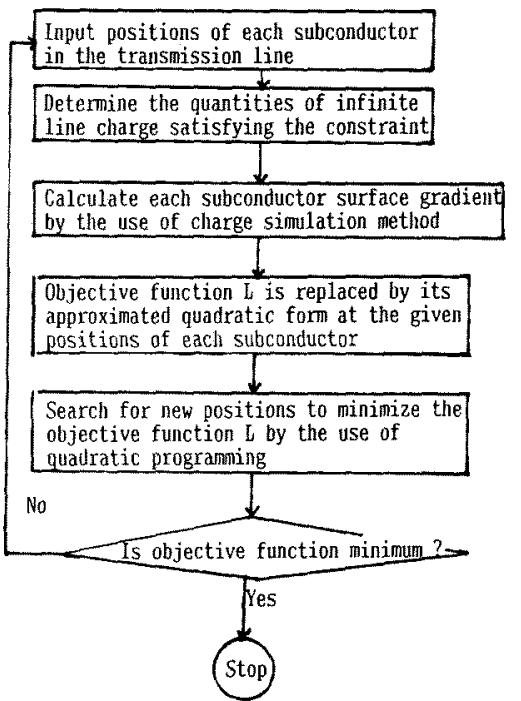

Fig. 4. Simplified flow chart.

$y_{i}: Y$ coordinate of contour points

$X_{j}: X$ coordinate of the infinite line charge points

$Y_{j}: Y$ coordinate of the infinite line charge points

\section{Constraint :}

$[P][Q]=[V]$

where

$[P]$ : Maxwell potential coefficient matrix $(s \times$ $s$ matrix)

$[Q]$ : infinite line charge quatity $\left(Q_{j R}+j Q_{j i m}, s\right.$ $\times 1$ vector)

$[V]:$ potential of contour point $\left(V_{i R}+j V_{i i m}, s \times\right.$ 1 vector)

The unknown variables in the above objective function $L$ are the center points of each subconductor (infinite line charge point: 76 variable $(X$ coordinate: $38, Y$ coordinate : 38) ] and the complex quantities of the infinite line charges (76 variable (real quantity: 38 , imaginary quantity : 38 ) ). 
But it is hard to minimize the objective function with these all unknown variables. Even though this problem can be solved, it is not possible to build the transmission line like the solution. Therefore the following items are assumed.

(1) The subconductor diameter is fixed.

(2) The air clearance of phase-to-phase (HA), the clearance between the phase conductor and ground $(\mathrm{HC})$, and the clearance between the overhead ground wire and the phase conductor $(\mathrm{HB})$ in the Fig. 2 are fixed because they are determined in designing the air clearance.

(3) As shown in Fig. 3, configuration of the phase conductor is identical to each other and arrangement of the subconductors is symmetric to $Y$ axis.

The method for solving this is shown in Fig. 4. If the initial positions of each subconductor are given, the quantities of infinite line charges satisfying the constraint can be determined. Based on these data, positions minimizing the objective function can be searched by the use of nonlinear successive quadratic programming. This procedure is repeated until the solution satisfies the Kuhn-Tucker condition. Maximum potential error in this method was $0.5 \%$ at calculation point of subconductor surface.

\section{Application result}

The method proposed in this paper is applied to two kinds of conductor bundle in $765 \mathrm{kV}$ three-phase double circuit transmission line.

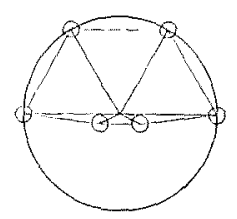

Fig. 5. Example of moving lower 2 subconductors only.

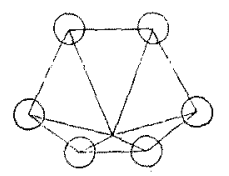

Fig. 6. Example of movine 6 subconductors all.
The first optimization type is to move the lower two subcondoctors only with the upper and middle four subconductors fixed as shown in Fig. 5. The second optimization type is to move six subconductors all as shown in Fig. 6.

Application result is shown in Fig. 7. The electric field distribution of each conductor bundle surface is also shown in Fig. 8. In the electric field of upper subconductor, GE asymmetric conductor bundle has the highest value and the asymmetric conductor bundle proposed in this paper (the lower two sub. conductor only moved: hereinafter it is called first type) is similar to the symmetric conductor bundle. The asymmetric conductor bundle proposed in this paper (all six subconductor moved: hereinafter it is called second type) has the lowest value and the electric field in a part of hanging water drops decreases especially. The range of the part of hanging water drops is $30^{\circ}$ for aged conductor as

Jait : cm
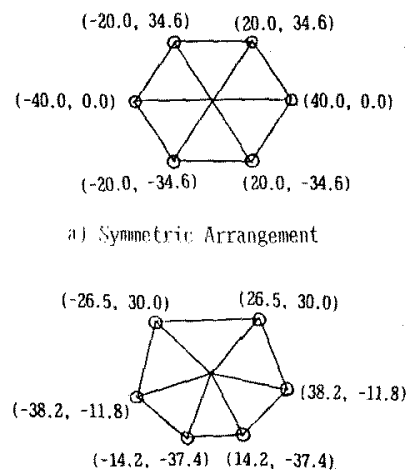

b) fir Asymmetric Arrangement $(\mathrm{Eo}=2)$

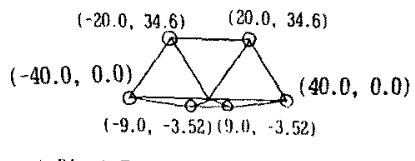

c) Eirst Type Asymmetric Arrangement

$$
(-18.5,4.98) \text { (-9.3.23.7) }(9.3,23.7)
$$

(1) Second Type Asymetric Arrangement

Fig. 7. Configuration of asymmetric arrangement proposed in this paper, symmetric arrangement and GE asymmetric arrangement. 


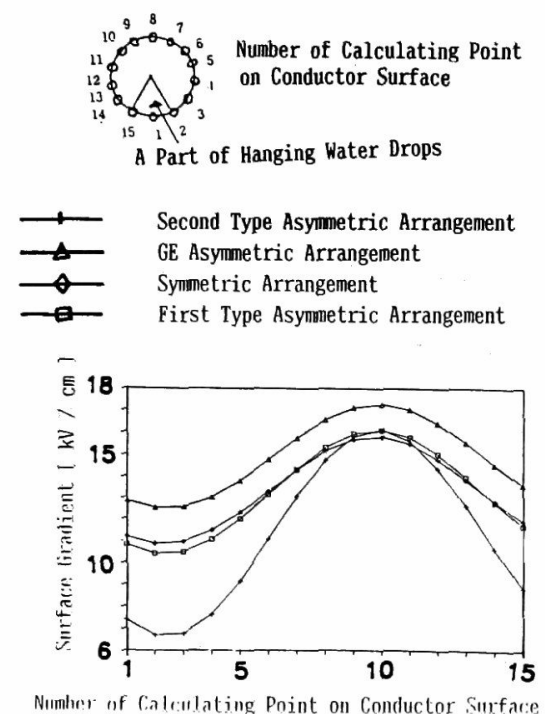

(a) Upper subconductor.

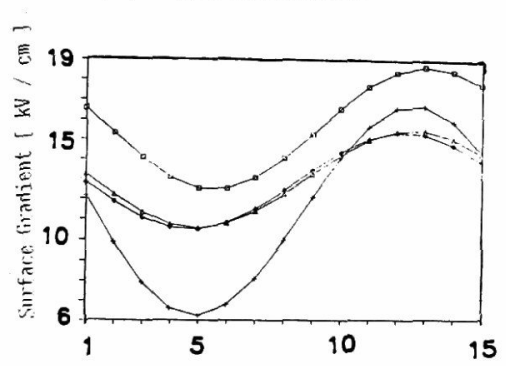

Nunher of lin lealating Point on Conductor surface

(b) Middle subconductor.

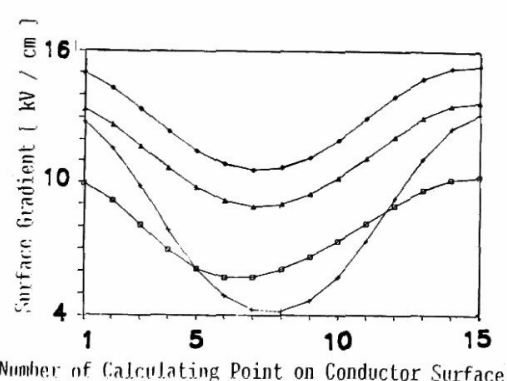

(c) Lower subconductor.

Fig. 8. Conductor surface gradient of asymmetric arrangement proposed in this paper, symmetric arraugement and GE asymmetric arrangement.

shown in Fig. 8. The hanging pattern of water droplet for new conductor is different from aged conductor ${ }^{(11)}$.

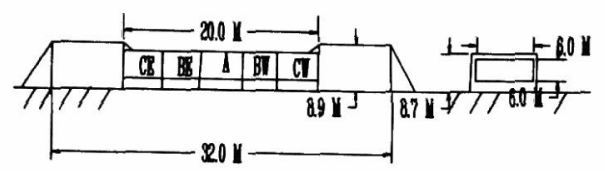

Fig. 9. Corona gage configuration.

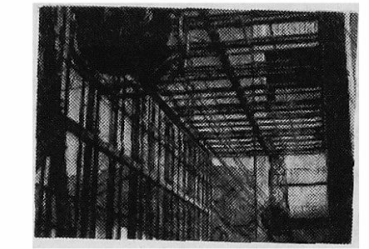

(a) GE asymmetric arrangement.

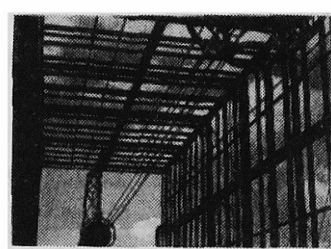

(b) Second type asymmetric arrangement.
Fig. 10. View of test equipment.

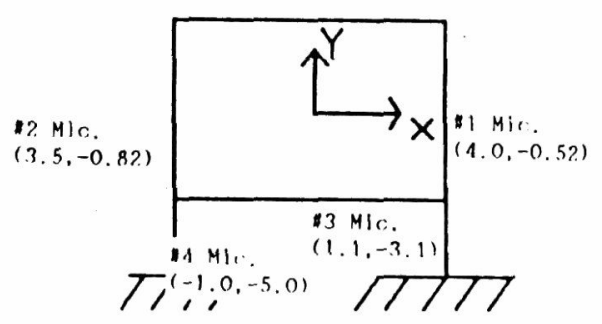

Fig. 11. Microphone position.

In the electric field of middle subconductor, the first type has the highest value and $\mathrm{GE}$ asymmetric conductor bundle is similar to the symmetric conductor bundle. The second type has the lowest value.

The electric field of lower subconductor decreases in this order; symmetric conductor bundle, GE asymmetric conductor bundle, the second type, the first type. Table 1 shows the average surface gradient of phase conductor ( $6 \times$ Rail, diameter of Rail : $2.96 \mathrm{~cm}$ ) and this value decreases in this order; symmetric conductor bundle, GE asymmetric conductor bundle, the first type, the second type.

\section{Test result}

The audible noise and radio interference of the above conductor bundle are measured in the $6 \mathrm{~m} \times 6$ $\mathrm{m} \times 20 \mathrm{~m}$ corona cage as shown in Fig. $9^{(10)}$. According to the rain rate, corona noise at water spray of $28 \mathrm{~mm} / \mathrm{hr}$ rain rate is referred to as "heavy rain 


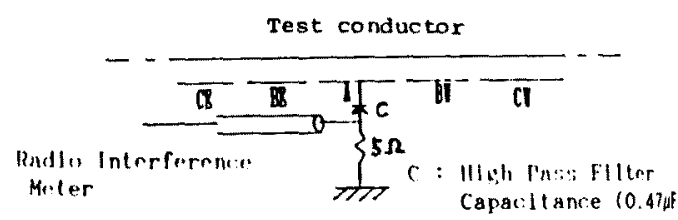

Fig. 12. Radio interference measuring circuit.

level". After the heavy rain level measurement is completed, corona noise level is also measured at 1 , 1.5 , and 2 minutes after water spray is stopped. The average of these values is referred to as "wet conductor level" like GE Project UHV and is assumed to be the same as $L_{50}$ (the sound level exceeded $50 \%$ of the measurement time) in the natural rain.

Fig. 10 is the view of test equipment. 4 condenser microphone ( $1 / 2$ inch diameter), sound level meter by Rion Co. NA-40, and a real time spectrum analyzer model SA-25 were used for AN measurement. AN was measured at the position as Fig. 11. ALTECH model NM 17/24 for RI measurement was used and radio noise voltage was measured under the CISPR specification at cage section $A$ insulated from the ground as Fig. 12. The frequency characteristics of $5 \Omega$ terminal voltage in Fig. 12 have been obtained. From this measurement, a $475 \mathrm{kHz}$ measuring frequency has been selected to meet the conditions that the received voltage should be stable with respect to a change in frequency and almost no interference due to external radio waves should be expected. The AN and RI measured in the cage are converted into the values generated from an infinite conductor bundle. As the measured value in the cage means the corona noise generated from the specific short single phase conductor bundle $(20 \mathrm{~m}$ in this paper), this value should be converted into the acoustic power at $1 \mathrm{~m}$ from an infinite conductor bundle.

$$
\begin{aligned}
P_{1}= & P_{\text {CAgE }}+10 \log R \\
& +10 \log \left[\pi /\left\{2 \tan ^{-1}(L / 2 R)\right\}\right]
\end{aligned}
$$

where

$P_{3}$ : acoustic power at $1 \mathrm{~m}$ from an infinite line

$P_{\text {CAGE }}$ : acoustic power measured in the corona cage

$R$ : distance from test conductor to microphone position

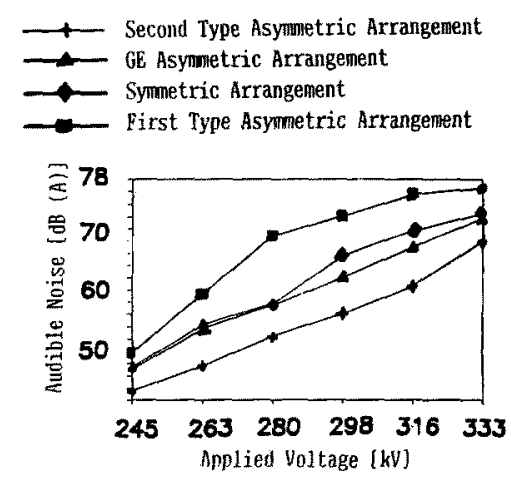

(a) Wet condutor level.

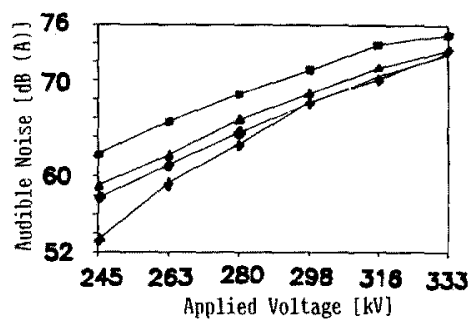

(b) Heavy rain level.

Fig. 13. Audible noise characteristics of asymmetric arrangement proposed in this paper in comparison with symmetric and $\mathrm{GE}$ asymmetric arrangement.

Table 1. Average surface gradient of phase conductor $(6 \times$ rail) for $765 \mathrm{kV}$ three-phase

\begin{tabular}{|c|c|c|c|c|}
\hline & & & \multicolumn{2}{|c|}{ unit $: \mathrm{kV} / \mathrm{cm}$} \\
\hline $\begin{array}{c}\text { Name } \\
\text { of } \\
\text { phase }\end{array}$ & $\begin{array}{l}\text { Symmetric } \\
\text { conductor } \\
\text { bundle }\end{array}$ & $\begin{array}{l}\text { Asymmetric } \\
\text { conductor } \\
\text { bundile by } \\
\mathrm{GE}\end{array}$ & $\begin{array}{l}\text { First type } \\
\text { proposed } \\
\text { in this } \\
\text { paper }\end{array}$ & $\begin{array}{l}\text { Second type } \\
\text { proposed } \\
\text { in this } \\
\text { paper }\end{array}$ \\
\hline $\begin{array}{l}P H-1 A \\
P H-2 C\end{array}$ & 13.307 & 13.282 & 12.5 & 10.614 \\
\hline $\begin{array}{l}\text { PH-1 B } \\
\text { PH-2 B }\end{array}$ & 12.347 & 12.330 & 11.59 & 9.873 \\
\hline $\begin{array}{l}\mathrm{PH}-1 \mathrm{C} \\
\mathrm{PH}-2 \mathrm{~A}\end{array}$ & 12.952 & 12.936 & 12,16 & 10.36 \\
\hline
\end{tabular}
double circuit transmission line.

$L$ : the length of a test conductor

The RI voltage measured in the cage is also converted into $\Gamma_{r}$ (the current per unit length of infinite line).

$$
\Gamma_{r}\left(d B_{\text {ref }} 1 \mu_{A} / \sqrt{m}\right)=V_{r}\left(d B_{\text {ref }} 1 \mu_{A}\right)-11.75
$$



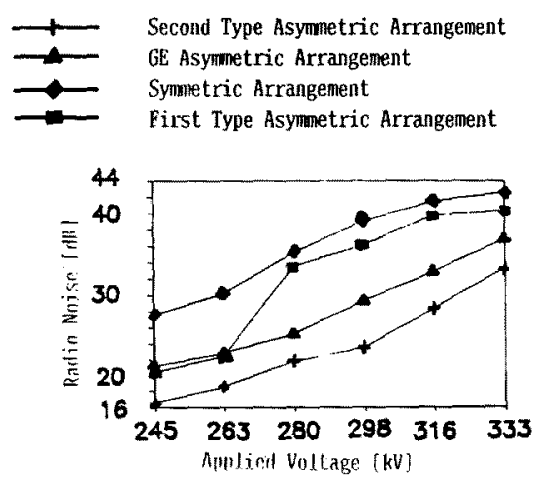

(a) Wet conductor level.

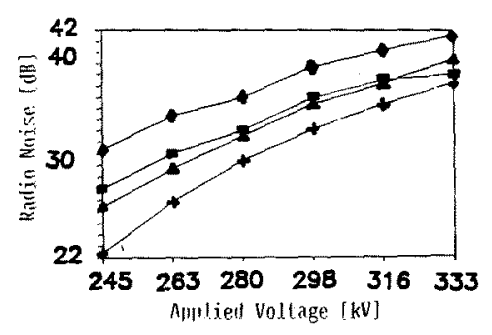

(b) Heavy rain level.

Fig. 14. Radio Interference characteristics of asymmetric arrangement proposed in this paper in comparison with symmetric and $G E$ asymmetric arrangement.

\section{where}

$V_{r}:$ RI voltage measured in the cage

The test result is shown in Figs 13 and 14. In case of applying the same voltage to the test conductor bundle in the cage, Fig. 13 shows that the second type generates the minimum AN. This result proves that the AN is minimized by the method proposed in this paper.

Meanwhile, Fig. 13 shows that the first type generates the maximum AN. This means that the AN increases when the specific subconductor surface gradient is excessively high, even though the average surface gradient of conductor bundle is low.

Therefore, to optimize the conductor bundle in view of corona noise, the average surface gradient of conductor bundle should be reduced while maintaining balanced surface gradient among subconductors. Fig. 14 shows that the Rl also has the similar characteristics to AN.

\section{Conclusion}

To reduce the corona noise in the E.H.V. AC transmission line, in this paper, a new asymmetric conductor bundle to decrease each subconductor surface gradient is determined. The charge simulation method is used to calculate the conductor surface gradient and the nonlinear successive quadratic programming is applied to find the optimal arrangement of subconductor. Through the analytical comparison and the corona cage test, it is proved that the asymmetric conductor bundle proposed in this paper can more reduce the average surface gradient of the phase conductor and the corona noise than the asymmetric conductor bundle proposed by $\mathrm{GE}$ as well as the conventional symmetric ones.

Based on this study result, to reduce the corona noise in transmission line, it is found that minimizing each subconductor surface gradient while maintaining balanced surface gradient among subconductors is more effective than equalizing the corona noise of each subconductor like GE.

(Manuscript received Feb. 25, 1991 reviced May 7, 1991)

\section{Reference}

(1) Transmission Line Reference Book $345 \mathrm{kV}$ and Above/ Second Edition Electric Power Research Institute(1982)

(2) M. Fukushima, Y.Sawada, et al.: "Audible Noise and Radio Noise from UHV AC Transmission Lines (Vil)- Measurement of Corona Performance of Conductor Bundles with a UHV Corona Cage", Central Research Institute Electric Power Industry, Japan (1978)

(3) A. C. Britten, E. G. Clarke \& H. E. Konkel: "Radio Interference, Corona Losses, Audible Noise and Power Fre quency Electric Fields as Factors in the Design of Escom's $765 \mathrm{kV}$ Lines", An Official Journal of the South African Institute of Electrical Engineers-ELECTRON, p. 7 (1988)

(4) M. G. Comber \& L. E. Zaffanella: "Audible Noise Reduc. tion by Bunble Geometry Optimization", IEEE Trans. Power Apparatus Syst., PAS-92, 1782 (1973)

(5) H. N. Scherer, Jr. \& G.S. Vassell: "Experience with the AEP $765 \mathrm{kV}$ System-Part 1: Overview", IEEE Power Engineering Society 1972 Winter Meeting and R\&D Conference

(6) M.G. Comber \& R. J. Nigbor: "Audible Noise Performance of Regular Asymmetric Bundles and effect of Conductor Aging on Project UHV's Three Phase Test Line", IEEE Trans Power Apparatis Syst., PAS-98, 561 (1979)

(7) G. Reklaitis, A. Ravindran \& K. M. Ragsdell : Engineer ing Optimization/Methods and Applications, p. 438 (1983) John Wiley \& Sons, Inc. 
(8) IMSL User's Manual-MATH/LIBRARY, Vol. 3, 895 (1987) IMSL, Inc.

(9) H. Singer, H. Steinbigler \& P. Wei $\beta$ : "A Charge Simulation Method for the Calculation of High Voltage Field", IEEE Trans. Power Apparatus Syst., PAS-93, 1660 (1974)

(10) S. W. Min, J. K. Park, et al.: "Audible Noise Level in Future $765 \mathrm{kV}$ Double Circuit Transmission Line of Korea Electric Power Corporation", Proc. 1988 U.S.-Japan Semi. nar on Electromagnetic Interference in Highly Advanced Social Systems (Modeling, Chracterization, Evaluation and Protection), p. 5.12 (1988)

(11) J. R. Booker, "Natural Aging of Non-Energized Aluminum Conductors", IEEE/PES 1986 Winter Meeting, 86 WM 1580 (1986)

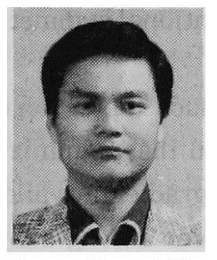

\section{Jong Keun Park (Member)}

Jong Keun Park was born in Chung-nam Province, Korea on October 21, 1952. He received BS degree in Electrical Engineering from Seoul National University, Korea in 1973, and $\mathrm{MS}$ and $\mathrm{Ph} . \mathrm{D}$. degree in Electrical Engineering from University of Tokyo, Japan in 1979 and 1982 respectively. He has been professor of Seoul National University since 1983. His main activities have been in calculation of harmonies in power system and electric field optimization.

Dr. Park is a member of the Institute of Electrical Engineers of Japan and the Institute of Electrical Engineers of Korea.

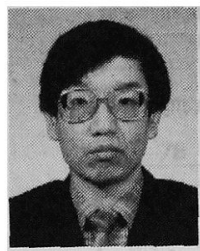

Suk Won Min (Non-member)

Suk Won Min was born in Seoul, Korea on January 3, 1958. He received $\mathrm{BS}, \mathrm{MS}$ and $\mathrm{Ph}$. D. degree in Electrical Engineering from Seoul National University in 1981, 1984 and 1991 respectively. He has been worked for Korea Electric Power Corporation from 1984 to 1991. He has been a Professor of Soon Chun Hyang University since 1991. His main activities have been in corona \& field effects and transient phenomena in power system.

Dr. Min is a member of the Institute of Electrical Engineers of Korea.

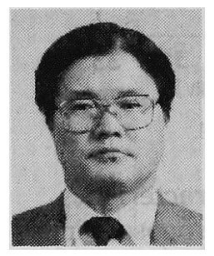

Jeong Boo Kim (Non-member)

Jeong Boo Kim was born in Kyung-book Province, Korea on November 14, 1943. He received BS, MS and Ph. D. degree in Electrical Engineering from Seoul National University in 1971, 1985 and 1990 respectively. In 1971, he joined Korea Electric Power Corporation. He has engaged in the study on insulation coordination, reactive power planning, transmission line design and corona \& field effects.

Dr. Kim is a member of the Institute of Electrical Engineers of Korea and IEEE. 